\title{
Mensuração diamétrica em um fragmento de Floresta Estacional Decidual
}

\author{
Anna Luiza Araújo Medeiros ${ }^{1}$ Ageu da Silva Monteiro Freire $\mathbb{(}^{2^{*}}$ Adriana Figueredo da Silva $\mathbb{0}^{1}$ \\ Amanda Brito da Silva $\mathbb{1}^{1}$ Yasmim Borges Câmara $\mathbb{1}^{1}$ Talvanis Clovis Santos de Melo $\mathbb{C}^{1}$ Juliana \\ Lorensi do Canto $\mathbb{1}^{1}$
}

${ }^{1}$ Universidade Federal do Rio Grande do Norte, RN 160 Km 03, Distrito de Jundiaí, Escola Agrícola de Jundiaí, CEP 59280000, Macaíba, RN, Brasil.

${ }^{2}$ Universidade Federal do Paraná, Av. Pref. Lothário Meissner, 632, Jardim Botânico, Campus III, CEP 80210-170, Curitiba, PR, Brasil

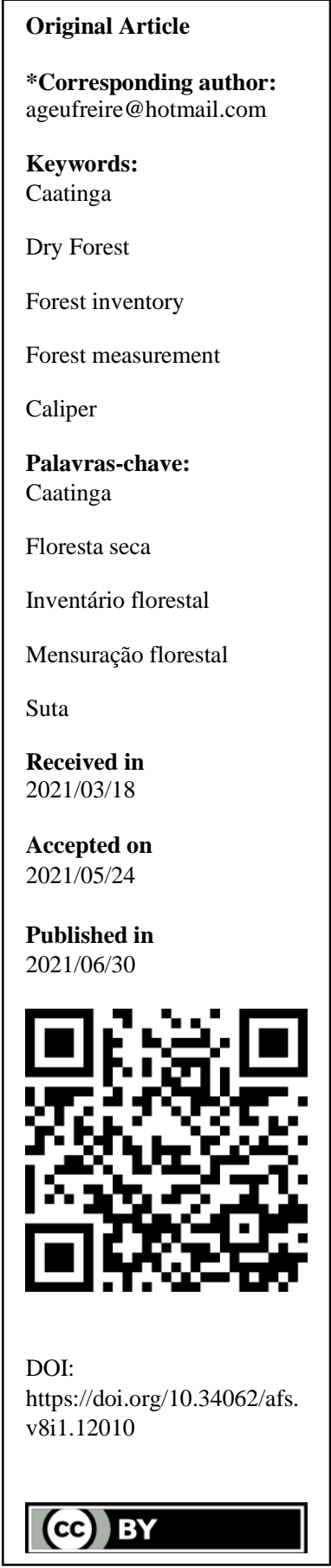

RESUMO: Com vegetação típica da região Nordeste do Brasil, a Caatinga é uma das maiores áreas de formação de florestas secas da América do Sul, justificando a busca por técnicas e instrumentos de mensuração que promovam maior agilidade e menor erro em inventários florestais. O objetivo do estudo foi comparar o desempenho e eficiência da fita métrica, da suta métrica mecânica e da suta finlandesa na medição diamétrica de árvores em um fragmento de Floresta Estacional Decidual. Em uma parcela amostral de $400 \mathrm{~m}^{2}$ foram medidos os Diâmetros a Altura do Peito de 62 árvores contidas nela, por cinco operadores, utilizando a fita métrica, a suta métrica mecânica e a suta finlandesa. Para cada medição houve a cronometragem do tempo gasto em cada árvore e do tempo total gasto na parcela. Após as medições, os operadores responderam a um questionário específico. A média do tempo de medição do DAP dos fustes das árvores foi maior com a fita métrica, seguida da suta métrica e da suta finlandesa, indicando que esta última fornece maior rapidez na medição. Não houve diferença nos valores de DAP medidos com os três instrumentos, mostrando que os mesmos dão resultados precisos. O operador influencia no tempo de medição, sendo necessário que os mesmos possuam conhecimento prévio sobre o manuseio e os procedimentos de medição. $\mathrm{O}$ estudo mostrou que a suta finlandesa foi o instrumento que forneceu maior rapidez. Os três instrumentos são eficientes para Caatinga, podendo ser usados em outras vegetações parecidas, como encontradas em florestas secas com outras localizações.

\section{Diametric measurement in a fragment of Seasonal Deciduous Forest}

ABSTRACT: With typical vegetation of the Northeast region of Brazil, the Caatinga is one of the largest areas of dry forest formation in South America, and it is necessary to seek techniques and instruments for measuring trees that promote greater agility and less error. The objective of the study was to compare the performance and efficiency of the tape measure, the Caliper and the Finnish Parabolic Caliper in the diametric measurement of trees in an area of Decidual Seasonal Forest. In a plot of $400 \mathrm{~m}^{2}, 62$ trees were selected. The diameter at breast height measurement was carried out by five operators, using the tape measure, caliper and Finnish Parabolic Caliper. For each measurement there was the timing of the time spent for the measurement in each tree and the total time spent in the plot. A questionnaire was also applied to the operators to evaluate the efficiency, advantages and disadvantages of each instrument. The average time of the DAP measurement of the tree fust was greater with the tape measure, followed by the caliper and the Finnish Parabolic Caliper, indicating that the latter provides faster measurement. It was also observed that there is no difference in the DAP size between the three instruments, showing that they give accurate results. The operator influences the measurement time, and it is necessary that they have previous knowledge about handling and measurement procedures. The study showed that Finnish Parabolic Caliper was the instrument that provided the fastest measurement. The three instruments are efficient for Caatinga and can also be used in other similar vegetation, as found in dry forests with other locations. 


\section{Medeiros et al.}

\section{Introducão}

O mundo possui extensas áreas de florestas secas, sendo que $94 \%$ dessas áreas localizam-se nos trópicos e no geral são menos ricas em espécies do que as florestas úmidas, consideradas também como ecossistemas frágeis e desprotegidos (Schwartz 2013). Praticamente todas as florestas secas estão expostas a uma variedade de ameaças, principalmente decorrentes das atividades humanas, necessitando de mais medidas de proteção (Miles et al. 2006). Além disso, Sunderland et al. (2015) explicam que há carência de pesquisas e pouca prioridade em políticas nacionais e internacionais em regiões com essas florestas, sendo necessário dentre tantas medidas para o conhecimento desses locais, a implementação de inventários das espécies, servindo para avaliação florística e biogeográfica, como também para estimar o estoque de carbono e o desenvolvimento das árvores.

Nas florestas secas da América Latina, os estudos são mais voltados aos impactos do ser humano nelas, sendo importante pesquisas sobre o manejo de florestas de produção, visto que há grande utilização dos recursos florestais, como na Caatinga que é uma das maiores áreas de formação de vegetação seca na região (Blackie et al. 2014). A Caatinga é um bioma exclusivo do Brasil, compreendendo $10 \%$ do território nacional, com cerca de $845.000 \mathrm{~km}^{2}$, apresentando $42,9 \%$ de área com cobertura florestal e $7,7 \%$ protegida por Unidades de Conservação, além de possuir vegetação tipicamente composta por árvores baixas e arbustos que geralmente perdem folhas no período seco (MAPA 2009). Também, a Caatinga possui biodiversidade voltada para várias atividades econômicas, com potencial para uso sustentável, visto que, apresenta acelerado desmatamento, principalmente devido a exploração ilegal e insustentável de lenha para uso doméstico e industrial (MMA 2020).

O Rio Grande do Norte foi analisado por meio do Inventário Florestal Nacional, estimando-se que haja 39 milhões de $\mathrm{m}^{3}$ de madeira estocada nas vegetações naturais, tendo o estado uma área de $42 \%$ composta por essas vegetações, com 91,26\% correspondente a tipologia de Caatinga (Serviço Florestal Brasileiro 2018). Com isto, os equipamentos de mensuração são fundamentais para o conhecimento da vegetação, principalmente para locais com vegetação de Caatinga, em que a supressão é rotineira, como para extração de lenha para energia e outros fins. Além disso, a análise da vegetação é importante para o licenciamento ambiental, os quais, técnicos geralmente atuam em várias parcelas de determinadas áreas para obter dados sobre a vegetação local, necessitando de instrumentos que promovam maior rapidez e resultados precisos.

Para se ter conhecimento quantitativo e qualitativo dos recursos florestais é indispensável a prática de mensuração acurada (Corte et al. 2016), pois o conhecimento das variáveis dendrométricas é fundamental para o manejo florestal (Machado et al. 2015). O diâmetro à altura do peito (DAP), tomado a $1,30 \mathrm{~m}$ do solo, é uma das principais variáveis medidas em inventários florestais, sendo geralmente mensurado com fita métrica, sutas mecânicas ou digitais (Corte et al. 2016). Além desses instrumentos, há a suta finlandesa, formada por um arco parabólico graduado conforme a sua curvatura, menos utilizada, mas com grande potencial para a mensuração diamétrica em vegetação de Caatinga, onde predominam espécies com múltiplos fustes com espinhos e acúleos, que dificultam a tomada dessa variável importante.

Diante da vegetação típica da região Nordeste, muitas vezes também caracterizada por uma alta densidade de fustes, é necessário buscar técnicas e instrumentos que promovam maior agilidade na medição e menor erro. Assim, o objetivo deste estudo foi comparar o desempenho e eficiência da fita métrica, da suta métrica e da suta finlandesa na medição diamétrica de árvores em uma área de Floresta Estacional Decidual, respondendo os seguintes questionamentos: i) Qual instrumento apresenta maior agilidade na medição? ii) $\mathrm{O}$ tamanho do fuste influencia o uso dos instrumentos? iii) O operador influencia o tempo de medição? iv) Qual instrumento apresenta maior precisão na obtenção de dados?

\section{Material e Métodos}

$\mathrm{O}$ trabalho foi realizado em um fragmento de Floresta Estacional Decidual de Terras Baixas (IBGE 1992), inserido em um ambiente de transição de Floresta Atlântica e Caatinga, predominando espécies deste último bioma. A área está localizada no município de Macaíba, Rio Grande do Norte $\left(5^{\circ}\right.$ $53^{\prime} \mathrm{S}$ e $35^{\circ} 23^{\prime} \mathrm{W}$ ) (Fig. 1). O local possui área com cerca de 270 ha e altitude média de $40 \mathrm{~m}$, apresentando clima do tipo As' e BSh' conforme classificação de Köppen, ocorrendo estação chuvosa entre os meses de maio e julho e estação seca entre os meses de setembro e dezembro (Alvares et al. 2013). A precipitação média anual é $1.086 \mathrm{~mm}$ (EMPARN 2017) e as árvores perdem mais de $80 \%$ de suas folhas em períodos desfavoráveis, onde no local é realizada exploração da madeira para estacas e mourões, como também uso de vegetação para ruminantes (Cestaro e Soares 2004). 


\section{Medeiros et al.}
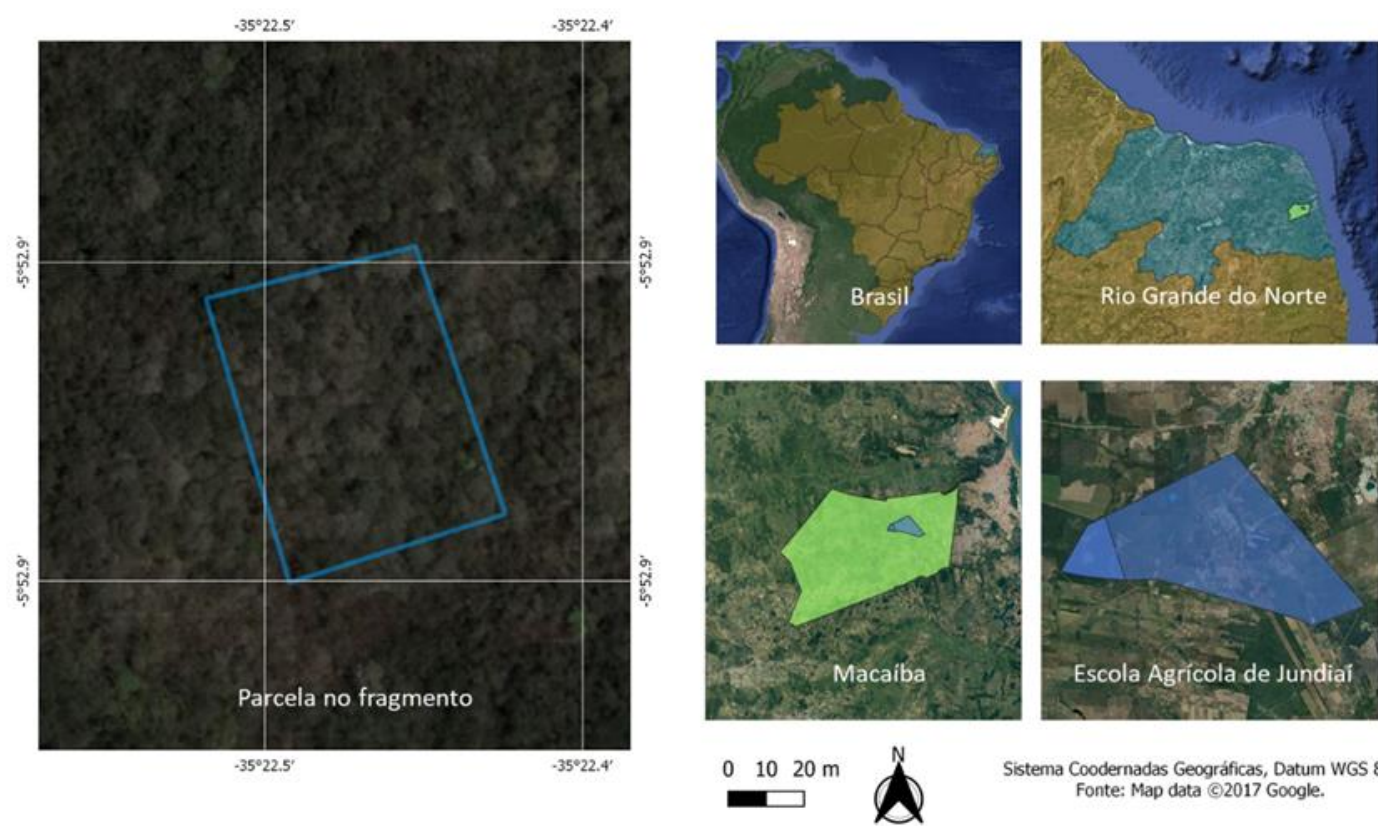

Sistema Coodernadas Geográficas, Datum WGS 84. Fonte: Map data (c) 2017 Google.

Figura 1. Localização geográfica da parcela no fragmento de Floresta Estacional Decidual na Escola Agrícola de Jundiaí, Macaíba, Rio Grande do Norte.

Foi demarcada uma parcela de $20 \mathrm{~m}$ x $20 \mathrm{~m}$ $\left(400 \mathrm{~m}^{2}\right)$ em uma área menos antropizada e com maior densidade de árvores no fragmento. A coleta de dados foi baseada no protocolo de medições de parcelas permanentes da rede de manejo florestal da Caatinga (Riegelhaupt et al. 2010). Foram selecionadas todas as árvores com Circunferência a Altura do Peito (CAP) $>6 \mathrm{~cm}$ e DAP $\geq 1,9 \mathrm{~cm}$, um total de 62, com a base do tronco dentro da parcela, mesmo estando com o fuste e copa para fora do alinhamento da parcela. As árvores foram identificadas com numeração (Tabela 1) e a mensuração foi realizada com três instrumentos, fita métrica, suta métrica mecânica e suta finlandesa (Figura 2).

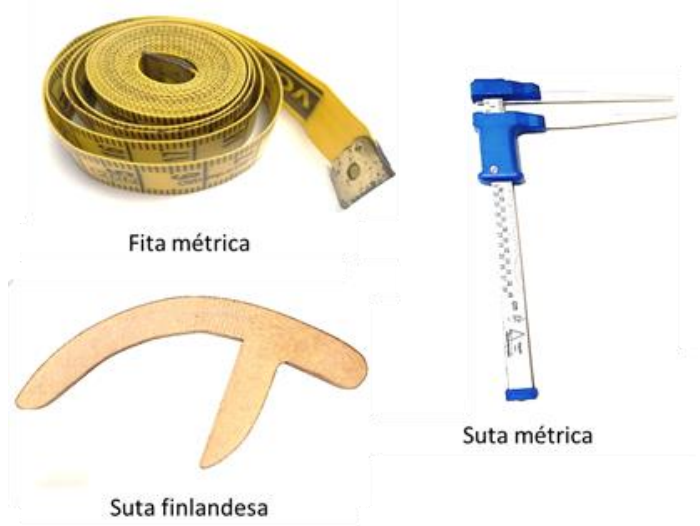

Figura 2. Fita métrica, suta métrica mecânica e suta finlandesa.

Tabela 1. Lista de espécies em parcela em um fragmento de Floresta Estacional Decidual no município de Macaíba, Rio Grande do Norte.

\begin{tabular}{ccc} 
Espécies & Família & Número de indivíduos \\
\hline Anacardium occidentale L. & Anacardiaceae & 4 \\
Anadenanthera colubrina (Vell.) Brenan & Fabaceae & 4 \\
Aspidosperma pyrifolium Mart. \& Zucc. & Apocynaceae & 2 \\
Cereus jamacaru DC. & Cactaceae & 2 \\
Cnidoscolus quercifolius Pohl & Euphorbiaceae & 4 \\
Cynophalla flexuosa (L.) J.Presl & Capparaceae & 4 \\
Handroanthus impetiginosus (Mart. ex DC.) Mattos & Bignoniaceae & 4 \\
Mimosa caesalpiniifolia Benth. & Fabaceae & 3 \\
Mimosa sp. & Fabaceaee & 13 \\
Mimosa tenuiflora (Willd.) Poir. & Fabaceae & 17 \\
Não identificados & & 62 \\
\hline Total & & \\
\hline
\end{tabular}




\section{Medeiros et al.}

A mensuração do DAP das árvores na parcela foi feita de forma individual e independente por cinco operadores, em que cada um tinha um assistente para anotar os dados. Cada operador usou um instrumento por vez e mediu todas as plantas. Para cada medição houve a cronometragem do tempo gasto pela aferição em cada árvore e o tempo total gasto na parcela, sendo este tempo anotado pelo assistente. Os dados coletados em campo foram submetidos ao programa estatístico BioEstat $5.0 \AA$ (Ayres et al. 2007), sendo realizada estatística descritiva para o DAP, tempo de medição das árvores e tempo de medição do operador. Foi realizado o teste de normalidade de Lilliefors, evidenciando distribuição não paramétrica nos dados, sendo feita a análise de variância de KruskalWallis em nível de 5\% de significância, entre os valores dos instrumentos e dos operadores, com comparação de médias pelo teste de Dunn quando ocorreu diferença significativa.

Após a obtenção dos dados, cada operador respondeu um questionário específico, com questões objetivas e subjetivas, avaliando a eficiência, vantagens e desvantagens de cada instrumento. Os questionários foram analisados no software Excel, versão 2013. As seguintes perguntas foram respondidas:

- Como você classifica a eficiência e rapidez da fita métrica, suta métrica mecânica e suta finlandesa?

- Como você classifica a praticidade de uso e manuseio da fita métrica, suta métrica mecânica e suta finlandesa?

- Como você classifica a praticidade de transporte em campo da fita métrica, suta métrica mecânica e suta finlandesa?
- Como você classifica a necessidade de treinamento e habilidade da fita métrica, suta métrica mecânica e suta finlandesa?

- Como você classifica a dificuldade de uso e manuseio da fita métrica, suta métrica mecânica e suta finlandesa?

- Como você classifica a facilidade de leitura da fita métrica, suta métrica mecânica e suta finlandesa?

- Qual a principal vantagem da fita métrica, suta métrica mecânica e suta finlandesa?

- Qual a principal desvantagem da fita métrica, suta métrica mecânica e suta finlandesa?

- De um modo geral, como você considera a fita métrica, suta métrica mecânica e suta finlandesa?

- Qual instrumento você prefere usar na Caatinga?

\section{Resultados e discussão}

A média do tempo de medição do DAP dos fustes das árvores foi maior com a fita métrica, seguida da suta métrica e da suta finlandesa, indicando que esta última fornece maior rapidez na medição (Tabela 2). Tal fato indica uma vantagem no uso dela, gastando menos tempo no trabalho. $\mathrm{O}$ erro padrão foi baixo para os três instrumentos, mostrando confiabilidade dos resultados e, o desvio padrão foi menor na suta finlandesa, evidenciando menor heterogeneidade nos dados em relação aos outros instrumentos. Isto evidencia mais uma vantagem frente aos outros instrumentos, em que a suta finlandesa já é muito usada na vegetação da Caatinga.

Tabela 2. Tempo de medição com fita métrica, suta métrica e suta finlandesa no DAP de árvores em um fragmento de Floresta Estacional Decidual.

\begin{tabular}{lccc} 
& Fita métrica & Suta métrica & Suta finlandesa \\
\hline Média (segundos) & $22,86 " \mathrm{c}$ & $19,67 " \mathrm{~b}$ & $14,87 " \mathrm{a}$ \\
Erro padrão & 1,08 & 1,26 & 0,70 \\
Desvio padrão & 24,42 & 28,92 & 15,23 \\
Tempo mínimo (segundos) & $2 "$ & $1 "$ & $1 "$ \\
Tempo máximo (segundos) & $126 "$ & $167 "$ & $168^{\prime \prime}$ \\
Coeficiente de variação & $106,84 \%$ & $147,01 \%$ & $102,39 \%$ \\
Tempo total na parcela (seg) & 39,6 & 36,2 & 33,2 \\
\hline
\end{tabular}

Letras iguais na linha não diferem estatisticamente entre si pelo teste de Dunn ao nível de $5 \%$ de probabilidade.

Com relação ao valor máximo de tempo de medição, a fita métrica apresentou o menor resultado (126"), podendo ser decorrente de árvores com fustes maiores, apresentando maior rapidez na medição. Além disso, o coeficiente de variação foi alto para os três instrumentos, expressando a alta variedade no tempo gasto para medição do DAP dos fustes, que pode ser pelas características da vegetação local, com alta variabilidade de diâmetro, influenciando o tempo de medição.
Para analisar o tempo gasto entre os operadores, os resultados indicaram que a suta métrica foi o instrumento que mais apresentou diferenças significativas, sendo a que tem maior variação de gasto de tempo entre quem as utiliza (Tabela 3). Tal fato, mostra a importância de se conhecer o instrumento antes de utilizá-lo, sendo importante treinamento principalmente para operadores iniciantes. Além disso, os resultados mostram que quem manuseia o instrumento também 


\section{Medeiros et al.}

Tabela 3. Comparação entre operadores no tempo de medição do DAP de árvores em um fragmento de Floresta Estacional Decidual.

\begin{tabular}{cccc}
\hline Operadores & Fita métrica & Suta métrica & Suta finlandesa \\
\hline $1 \times 2$ & $<0,05$ & $<0,05$ & $\mathrm{~ns}$ \\
$1 \times 3$ & $\mathrm{~ns}$ & $<0,05$ & $<0,05$ \\
$1 \times 4$ & $\mathrm{~ns}$ & $<0,05$ & $<0,05$ \\
$1 \times 5$ & $<0,05$ & $<0,05$ & $<0,05$ \\
$2 \times 3$ & $\mathrm{~ns}$ & $\mathrm{~ns}$ & $\mathrm{~ns}$ \\
$2 \times 4$ & $\mathrm{~ns}$ & $\mathrm{~ns}$ & $\mathrm{~ns}$ \\
$2 \times 5$ & $\mathrm{~ns}$ & $\mathrm{~ns}$ & $\mathrm{~ns}$ \\
$3 \times 4$ & $\mathrm{~ns}$ & $\mathrm{~ns}$ & $\mathrm{~ns}$ \\
$3 \times 5$ & $\mathrm{~ns}$ & $<0,05$ & $\mathrm{~ns}$ \\
\hline
\end{tabular}

Diferença significativa $(\mathrm{p}<0,05)$; Diferença não significativa (ns) pelo teste de Dunn.

influência no tempo gasto, em que, por exemplo, uma pessoa com mais experiência pode ter mais agilidade nas medições.

Os resultados referentes ao diâmetro dos fustes, indicaram que os valores foram estatisticamente iguais entre todos os operadores, não havendo também diferença estatística entre os instrumentos (Tabela 4). O coeficiente de variação mostra alta variabilidade no diâmetro dos fustes, comprovando que isto pode influenciar no tempo de medição. Com isto, observa-se que a fita métrica foi a que apresentou menor valor no tempo máximo gasto, sugerindo ser um instrumento eficiente para medição de fustes maiores. Além disso, todos os equipamentos mostraram precisão nos resultados, independente da agilidade dos operadores e do tempo gasto para medição.

Tabela 4. Média do DAP com fita métrica, suta métrica e suta finlandesa em árvores em um fragmento de Floresta Estacional Decidual.

\begin{tabular}{|c|c|c|c|c|c|c|}
\hline & & \multicolumn{5}{|c|}{ Operadores } \\
\hline & & 1 & 2 & 3 & 4 & 5 \\
\hline \multirow{4}{*}{ Fita métrica } & Média $(\mathrm{cm})$ & $6,22 \mathrm{aA}$ & $6,39 \mathrm{aA}$ & $6,51 \mathrm{aA}$ & $6,43 \mathrm{aA}$ & $6,25 \mathrm{aA}$ \\
\hline & Erro Padrão & 0,46 & 0,48 & 0,50 & 0,48 & 0,47 \\
\hline & Desvio padrão & 4,29 & 4,41 & 4,59 & 4,44 & 4,40 \\
\hline & $\begin{array}{l}\text { Coeficiente de } \\
\text { variação }\end{array}$ & 69,03 & 69,07 & 70,57 & 69,16 & 70,47 \\
\hline \multirow{4}{*}{ Suta métrica } & Média $(\mathrm{cm})$ & $6,03 \mathrm{aA}$ & $6,04 \mathrm{aA}$ & $6,06 \mathrm{aA}$ & $6,54 \mathrm{aA}$ & $6,17 \mathrm{aA}$ \\
\hline & Erro Padrão & 0,48 & 0,48 & 0,50 & 0,55 & 0,52 \\
\hline & Desvio padrão & 4,47 & 4,43 & 4,62 & 5,10 & 4,81 \\
\hline & $\begin{array}{c}\text { Coeficiente de } \\
\text { variação }\end{array}$ & 74,06 & 73,37 & 76,24 & 78,00 & 78,03 \\
\hline \multirow{4}{*}{ Suta finlandesa } & Média $(\mathrm{cm})$ & $5,68 \mathrm{aA}$ & $7,15 \mathrm{aA}$ & $5,65 \mathrm{aA}$ & $6,49 \mathrm{aA}$ & $5,88 \mathrm{aA}$ \\
\hline & Erro Padrão & 0,38 & 0,71 & 0,38 & 0,42 & 0,38 \\
\hline & Desvio padrão & 3,53 & 6,54 & 3,57 & 3,90 & 3,59 \\
\hline & $\begin{array}{c}\text { Coeficiente de } \\
\text { variação }(\%)\end{array}$ & 62,16 & 91,42 & 63,13 & 60,03 & 61,05 \\
\hline
\end{tabular}

Letras minúsculas iguais na linha e maiúsculas iguais na coluna não diferem estatisticamente entre si pelo teste de Dunn ao nível de 5\% de probabilidade.

$\mathrm{Na}$ avaliação qualitativa dos operadores, a maioria respondeu que a fita métrica apresenta eficiência e rapidez moderada, enquanto maior parte respondeu que essas atribuições foram alta e muito alta para a suta métrica e suta finlandesa, respectivamente (Tabela 5). Com relação a praticidade de uso e manuseio a fita métrica e a suta finlandesa foram as que tiveram respostas mais positiva. Já para a praticidade de transporte em campo, a fita métrica foi o melhor instrumento, como também, foi visto pelos operadores que ela foi a que menos precisa de treinamento e habilidade e a que apresenta menor dificuldade no uso e manuseio. Para facilidade de leitura, a suta finlandesa foi a única que apresentou valor negativo, sendo suta métrica o instrumento com maior avaliação positiva.

Tabela 5. Avaliação qualitativa dos operadores referentes a utilização da fita métrica, suta métrica e suta finlandesa na medição diamétrica de árvores em um fragmento de Floresta Estacional Decidual. 1=Muito alta; 2=Alta; 3=Moderada; e 4=baixa. 


\begin{tabular}{|c|c|c|c|c|c|c|c|c|c|c|c|c|}
\hline & \multicolumn{4}{|c|}{ Fita métrica } & \multicolumn{4}{|c|}{ Suta métrica } & \multicolumn{4}{|c|}{ Suta finlandesa } \\
\hline & 1 & 2 & 3 & 4 & 1 & 2 & 3 & 4 & 1 & 2 & 3 & 4 \\
\hline Eficiência e rapidez & - & $40 \%$ & $60 \%$ & - & - & $60 \%$ & $40 \%$ & - & $60 \%$ & $60 \%$ & - & - \\
\hline $\begin{array}{l}\text { Praticidade de uso e } \\
\text { manuseio }\end{array}$ & $40 \%$ & - & $40 \%$ & $20 \%$ & - & $40 \%$ & $60 \%$ & - & $40 \%$ & $60 \%$ & - & - \\
\hline $\begin{array}{c}\text { Praticidade no transporte } \\
\text { em campo }\end{array}$ & $100 \%$ & - & - & - & - & - & $40 \%$ & $60 \%$ & - & $80 \%$ & $20 \%$ & - \\
\hline $\begin{array}{c}\text { Necessidade de } \\
\text { treinamento e habilidade }\end{array}$ & - & - & $20 \%$ & $80 \%$ & - & $20 \%$ & $60 \%$ & $20 \%$ & - & $40 \%$ & $40 \%$ & $20 \%$ \\
\hline $\begin{array}{l}\text { Dificuldade no uso e } \\
\text { manuseio }\end{array}$ & - & - & - & $100 \%$ & - & - & $80 \%$ & $20 \%$ & - & - & $40 \%$ & $60 \%$ \\
\hline Facilidade de leitura & $40 \%$ & $40 \%$ & $20 \%$ & - & $60 \%$ & $20 \%$ & $20 \%$ & - & - & $40 \%$ & $40 \%$ & $20 \%$ \\
\hline
\end{tabular}

Os operadores também avaliaram que as principais vantagens da fita métrica era a praticidade no manuseio e no transporte em campo, já a suta métrica tem mais vantagem na precisão e visualização das medidas e, a suta finlandesa é mais prática na medição. Em contrapartida, a principal desvantagem da fita métrica é que demora mais para medir e visualizar os dados em comparação aos outros instrumentos. Também, foi avaliado que a principal desvantagem da suta métrica é o transporte em campo e da suta finlandesa é a visualização da graduação. De um modo geral, os operadores consideraram a suta métrica melhor para medição da vegetação do local, em que os demais instrumentos também apresentaram boa avaliação.

A suta finlandesa é o instrumento que fornece maior rapidez, o qual o operador poderá fazer o trabalho em menor tempo. Porém, a sua principal limitação está relacionada ao tamanho diamétrico de fustes maiores, em que a fita métrica seria o instrumento mais adequado, pois a suta finlandesa geralmente não possui maiores graduações. Já a suta métrica mostra atribuições intermediárias entre a fita métrica e a suta finlandesa. Contudo, diante da precisão nos resultados dos instrumentos avaliados, observou-se que os três são adequados para mensurações em campo. Com isto, cabe avaliar a fisionomia da vegetação, escolhendo o instrumento conforme tamanho do fuste, ou até mesmo o operador utilizar a suta finlandesa para árvores de fustes menores e a fita métrica para árvores de fustes maiores, já que a mesma é maleável e fácil de transportar.

Ademais, cabe ao operador definir qual o instrumento é mais adequado para seu manuseio, sendo importante que o mesmo tenha conhecimento prévio de como utilizar, pois foi constatado que o profissional que usará os instrumentos influenciará o tempo de medição. Vale salientar que isto demonstra as limitações impostas aos seres humanos e por se tratar de um ambiente com baixa precipitação, alta radiação solar e vegetação seca, o operador deve seguir protocolos para sua saúde e segurança. Schettino et al. (2016) observaram que a sobrecarga térmica em atividades de mensuração florestal impacta os trabalhadores, sendo fundamental tomar medidas organizações para atenuar a exposição a esse fator ambiental, contribuindo para saúde do operador e consequentemente para a produtividade.

Nos trabalhos em que é necessário saber o DAP de plantas na Caatinga, a fita métrica e a suta finlandesa são os instrumentos com uso mais comum, não havendo na literatura quais são os mais eficientes para este tipo de vegetação. Porém, para espécies comerciais já se tem estudos, como um realizado em campo experimental da Escola Superior de Agricultura Luiz de Queiroz da Universidade de São Paulo, na qual foram usados equipamentos de aferição para o diâmetro (suta e a fita métrica) em 20 árvores de Eucalyptus spp e Pinus spp, apresentando valores estatisticamente semelhantes no diâmetro (Freitas e Wichert 1998). Tal resultado demonstra semelhança com o presente estudo em que os instrumentos forneceram diâmetros com valores estatisticamente iguais

A média de DAP também foi estatisticamente semelhante para medição com fita métrica e suta métrica na espécie Cryptomeria japonica, sendo constatado bom desempenho dos instrumentos (Corte et al. 2016). Porém Baptista e Martins (2017) concluíram que o instrumento mais preciso na medição do diâmetro de Eucalyptus spp com 34 anos de idade no diâmetro foi a suta métrica. Como visto no trabalho, o erro padrão foi próximo no DAP entre todos os operadores e os instrumentos, mostrando que todos são eficientes na obtenção de dados para a vegetação da Caatinga.

\section{Conclusões}

$\mathrm{O}$ estudo mostrou que a suta finlandesa foi o instrumento que forneceu maior rapidez na medição do fuste de vegetação da Caatinga, porém é limitado, não sendo eficaz em árvores com fustes maiores, sendo a fita métrica mais recomendada. Também foi observado que não há diferença no tamanho do DAP entre os três instrumentos, mostrando que os mesmos dão resultados precisos.

O operador influencia no tempo de medição, sendo necessário que os mesmos possuam conhecimento prévio sobre o manuseio e os procedimentos de medição. Também, sugere-se que os operadores vejam qual melhor instrumento para eles, assim como devem tomar medidas de conforto no trabalho para obter melhor desempenho e 
produtividade. No mais, os três instrumentos são eficientes para Caatinga e podem ser usados também em outras vegetações parecidas, como encontradas em florestas secas em outras localizações.

\section{Referências}

Alvares CA, Stape JL, Sentelhas, PC, Gonçalves J LM, Sparovek G (2013) Köppen's climate classification map for Brazil. Meteorologische Zeitschrift, 19(6), 711-728. doi: 10.1127/09412948/2013/0507

Ayres M, Ayres Júnior M, Ayres DL, Santos AS (2007) BioEstat: aplicações estatísticas nas áreas de ciências biométricas. Versão 5.0. Belém: Sociedade Civil Mamirauá, MCT-CNPq.

Baptista IS, Martins EP (2017) Comparação entre as medidas obtidas através do uso da suta, vara de biltmore e fita métrica em uma floresta plantada: a matemática está em tudo. In: $4^{\circ}$ Encontro de ciência e tecnologia, Rondônia.

Blackie R, Baldauf C, Gautier D, Gumbo D, Kassa H, Parthasarathy N, Paumgarten F, Sola P, Pulla S, Waeber P, Sunderland T (2014) Tropical dry forests: The state of global knowledge and recommendations for future research. Discussion Paper. Bogor, Indonesia: Center for International Forestry Research. 39p.

Cestaro LA, Soares JJ (2004) Variações florística e estrutural e relações fitogeográficas de um fragmento de floresta decídua no Rio Grande do Norte, Brasil. Acta Botanica Brasilica, 18(2), 203218. doi: 10.1590/S0102-33062004000200001

Corte APD, Oliveira KA, Coutinho VM, Sanquetta CR, Behling A (2016) Desempenho de diferentes equipamentos para mensuração de diâmetro a 1,30 $\mathrm{m}$, altura individual total, e volume do fuste em Cryptomeria japonica (Thunb. ex L. f.) D. Don.. Enciclopédia biosfera, 13, 432-441.

EMPARN (2017) Empresa de Pesquisa Agropecuária do Rio Grande do Norte. Disponível em: http://www.emparn.rn.gov.br/Index.asp. Acesso em 31 jul. 2017.

Freitas AG, Wichert MCP (1998) Comparação entre instrumentos tradicionais de medição de diâmetro e altura com o criterion 400. IPEF Circular Técnica, Piracicaba, Brasil: Instituto de Pesquisas e Estudos Florestais, (188), 1-7.
IBGE (2012) Manual Técnico da Vegetação Brasileira, $2^{\text {a }}$ edição. Rio de Janeiro: Instituto Brasileiro de Geografia e Estatística. 271p.

Machado SA, Souza RF, Aparecido LMT, Ribeiro A, Czelusniak BH (2015) Evolução das variáveis dendrométricas da bracatinga por classe de sítio. Cerne, 21, 199-207. doi: $10.1590 / 01047760201521021222$.

Miles L, Newton AC, DeFries RS, Ravilious C, May I, Blyth S, Kapos V, Gordon J (2006) A global overview of the conservation status of tropical dry forests. Journal of Biogeography, 33(3), 491-505. doi: 10.1111/j.1365-2699.2005.01424.x

MAPA (2019) Florestas do Brasil em resumo: 2019 , $1^{a}$ edição. Brasília: Ministério da Agricultura, Pecuária e Abastecimento/Serviço Florestal Brasileiro. 207p.

MMA (2020) Caatinga. Ministério do Meio Ambiente. Disponível em: https://www.mma.gov.br/biomas/caatinga. Acesso em 15 nov. 2020

Riegelhaupt EM, Pareyn FGC, Gariglio MA (2010) O manejo florestal como ferramenta para o uso sustentável e conservação da caatinga. In: Gariglio, M. A. et al. (Org.). Uso sustentável e conservação dos recursos florestais da caatinga. Brasília: Serviço Florestal Brasileiro. 349-367.

Schettino S, Minette LJ, Souza AL, Souza AP (2016) Avaliação ergonômica do processo de mensuração florestal. Scientia Forestalis, 44, 575-586. doi: 10.18671/scifor.v44n111.04

Schwartz MD (2013) Phenology: An integrative environmental science, $1^{\mathrm{a}}$ edição. Dordrecht Heidelberg New York London: Springer. 564p.

MMA (2018) Inventário Florestal Nacional Rio Grande do Norte, principais resultados. Série relatórios técnicos - IFN, Brasília: Ministério da Agricultura, Pecuária e Abastecimento/Serviço Florestal Brasileiro. 64p.

Sunderland TCH, Apgaua D, Baldauf C, Blackie R, Colfer C, Cunningham A, Dexter K, Djoudi H, Gautier D, Gumbo D, Ickowitz A, Kassa H, Parthasarathy N, Pennington T, Paumgarten F, Pulla S, Sola P, Tng D, Waeber P (2015) Global dry forests: a prologue. International Forestry Review, 17, 1-9. doi: 10.1505/146554815815834813 